\title{
Magnetic Resonance Appearance of Pulmonary Type of Primary Small Cell Carcinoma of the Ovary: A Case Report and Literature Review
}

\author{
Xuechao Du (iD) ${ }^{1}$, Pengtao Sun ${ }^{2}$, Yuan Zhao ${ }^{3}$, Yuchang Yan (iD ${ }^{1}$ and Zhenyu Pan (iD) ${ }^{1,}$ \\ ${ }^{1}$ Department of Radiology, Beijing Chaoyang Hospital, Capital Medical University, Beijing, China \\ ${ }^{2}$ Department of Radiology, Beijing Shijitan Hospital, Capital Medical University, Beijing, China \\ ${ }^{3}$ Department of Pathology, Beijing Chaoyang Hospital, Capital Medical University, Beijing, China \\ "Corresponding author: Department of Radiology, Beijing Chaoyang Hospital, Capital Medical University, Beijing, China. Email: panzhenyu@ccmu.edu.cn
}

Received 2020 May 04; Revised 2020 December 15; Accepted 2020 December 27.

\begin{abstract}
The pulmonary type of primary small cell carcinoma of the ovary (SCCOPT) is a rare aggressive malignancy with a poor prognosis, usually occurring in postmenopausal women. Few literatures have emphasized on the magnetic resonance (MR) imaging features. In this paper, we analyze its MR imaging findings in combination with pathological manifestations. We report a case of a 51-yearold woman who presented with abdominal pain and distension. Several tumor markers were elevated. MR scan of the pelvis was performed. It revealed a heterogeneous lobulated mass with solid and cystic components originating from the right adnexa. The patient underwent total abdominal hysterectomy with bilateral salpingo-oophorectomy. Histology showed a tumor comprising areas of classical small-cell carcinoma, and SCCOPT was diagnosed based on histopathology and immunohistochemistry. SCCOPT is a rare aggressive malignancy with certain characteristic imaging features. The solid component exhibits slightly higher signal on T2-weighted imaging with fat suppression, restricted diffusion on diffusion weighted imaging (DWI) and apparent diffusion coefficient (ADC) maps, and honeycomb-like persistent enhancement. More data are needed to better understand its specific imaging manifestations.
\end{abstract}

Keywords: Small Cell Carcinoma, Ovary, Pulmonary Type, Magnetic Resonance Imaging, Case Report

\section{Introduction}

Primary small cell carcinoma of the ovary (SCCO) was first described in 1979 (1). It is a highly aggressive malignancy with a poor prognosis, which accounts for less than $1 \%$ of all ovarian cancers $(2,3)$. World Health Organization (WHO) classified SCCO as miscellaneous tumors (2014 edition). Two types of primary SCCO have been described, the hypercalcemic type (SCCOHT) and the pulmonary type (SCCOPT), which are clinically and histopathologically distinct entities. Both of these tumors are very uncommon in the ovary and SCCOPT is extremely rare with approximately 24 cases reported in the English literature to date. The majority of SCCOPT occurred in mature cystic teratomas, with only nine cases being described as "pure" primary SCCOPT (4). SCCOPT often occurs in elderly women (2). Clinically, it has many similarities with ovarian epithelial cell carcinoma (5), and preoperative diagnosis is difficult. Currently, most of the literatures described its pathological characteristics, clinical treatment and prognosis, and little attention has been paid to the imaging manifestations. With the purpose of reaching better understand- ing of the disease, we report a case of unilateral primary SCCOPT, the magnetic resonance imaging (MRI) manifestations and clinical data, and further reviewed relevant literatures.

\section{Case Presentation}

A 51-year-old woman was admitted due to lower abdominal pain and distension. No vaginal irregular bleeding was found. She had a history of breast cancer, and received radical mastectomy five years ago, followed by postoperative chemotherapy and targeted therapy. She had no genetic family history.

Table 1 summarizes the patient's details including clinical data, imaging findings, pathological features and treatment. The serum cancer antigen 125 (CA125) level elevated to $68.79 \mathrm{U} / \mathrm{ML}$ (normal range 0 - 35.0 U/ML), cancer antigen 199 (CA 199) to $43.51 \mathrm{IU} / \mathrm{ML}$ (normal range 0 - $39.0 \mathrm{U} / \mathrm{ML}$ ) and carcinoembryonic antigen (CEA) to $15.87 \mathrm{ng} / \mathrm{mL}$ (normal range 0 - 5.0 U/ML). Ultrasound of the pelvis demonstrated a complex mass extending to the right adnexa. Hence, MRI of the pelvis was performed, which revealed a 
$6.1 \times 7.6 \times 8.0 \mathrm{~cm}$ heterogeneous lobulated mass with solid and cystic components originating from the right adnexa. The lesion had iso- to hypo-intense signal on T1-weighted imaging (T1WI) (Figure 1A). T2-weighted imaging with fat suppression (T2WI FS) (Figure 1B) and T2-weighted imaging (T2WI) (Figure 1E) showed the solid component had slightly higher heterogeneous signal intensity (SI), higher than the SI of uterine myometrium, with hypointense foci; and the cystic component had the same SI as water. The solid component showed hyperintense on diffusionweighted imaging (DWI) (Figure 1C) and hypointense on apparent diffusion coefficient (ADC) mapping (Figure 2D), with $\mathrm{ADC}$ values ranging from $0.77 \times 10^{-3} \mathrm{~s} / \mathrm{mm}^{2}$ to 0.95 $\times 10^{-3} \mathrm{~s} / \mathrm{mm}^{2}$. The border between the lesion and the uterus, the sigmoid colon and adjacent small intestine was unclear (Figure 1B), while its posterior margin was welldemarcated with T2-hypointense capsule. Moderate pelvic effusion was observed (Figure 1E), and the endometrium was slightly thickened (Figure $1 \mathrm{~F}$ ). After administration of contrast media, the solid component of the lesion showed honeycomb-like intense enhancement (Figure 2A and B). The time-intensity curve (TIC) of dynamic enhanced was fast-rising platform type. No enlarged lymph nodes were detected. Computer tomography (CT) of the chest and abdomen showed no evidence of metastasis. We therefore suspected ovarian cancer and performed total abdominal hysterectomy with bilateral salpingo-oophorectomy. During operation, we detected a right ovarian tumor, which was adherent to the posterior wall of the uterus, as well as the right broad ligament, the sigmoid colon, and the small intestine. A nodule was detected in the left pelvic peritoneum. Intraoperative blood loss was $1300 \mathrm{~mL}$.

Pathological examination revealed primary SCCOPT. Grossly, the right ovary measured $10.5 \times 7.0 \times 4.0 \mathrm{~cm}$ with lobulated margins. Microscopically (Figure 2C), a malignant solid neoplastic component was observed, which consisted of nests, trabeculae, and islands of closely packed small cells with scant cytoplasm, hyperchromatic nuclei, obvious cell atypia and mitotic figures. Tumor was separated by abundant fibrous tissue. Large necrosis area was also seen. Malignant tumors can be seen in the muscularis and adventitia of bilateral fallopian tubes, as well as in the peritoneal nodule. The uterus, intestine and lymph nodes (0/20) were not involved. Immunohistochemically, neoplastic cells expressed diffuse staining with neuroendocrine markers such as cluster of differentiation 56 (CD56) and synaptophysin. Chromogranin-A (CgA) and cytokeratin 20 (CK20) was focally positive. Ki67 index was approximately 50\%. The patient was assigned an International Federation of Gynecology and Obstetrics (FIGO) ovarian cancer stage of IIB.

She underwent three cycles of taxol-carboplatin chemotherapy (TC) and myelosuppression occurred. The fourth pre-chemotherapy assessment revealed multiple metastases in the lungs and abdomen, and the chemotherapy regimen was changed to liposomal doxorubicin hydrochloride (40 mg) and carboplatin (0.5 g). Unfortunately, the tumor continued to worsen, and the following fifth PAC chemotherapy regimen (cisplatin $100 \mathrm{mg}$, liposome doxorubicin $40 \mathrm{mg}$, and cyclophosphamide $0.8 \mathrm{~g}$ ) was also proved ineffective. Therefore, she received radiation therapy and genetic testing, which showed sensitivity to PARP (poly-ADP-ribose polymerase) inhibitors. Hence, she started to take oral olaparib tablets $300 \mathrm{mg}$ twice a day, and anlotinib hydrochloride capsules $12 \mathrm{mg} /$ day (two weeks for chemotherapy and one week for discontinuation, take medication periodically). For now, the patient has been followed up for 8 months after operation. During this period, she continued to lose weight, from $72 \mathrm{~kg}$ to $64 \mathrm{~kg}$. Regular postoperative CT follow-up showed gradual increase in both number and size of liver and lungs metastases, accompanied by gradual increase of the serum CEA and CA199.

\section{Discussion}

SCCO is an extremely rare and aggressive ovarian malignancy, which accounts for less than $1 \%$ of all ovarian neoplasms (2). It may be growing rapidly, and sometimes, the clinical history may be short. The majority of patients are beyond stage I at presentation (6), and only around $20 \%$ to $25 \%$ have disease confined to the ovary. Two types of primary SCCO have been described, the hypercalcemic type (SCCOHT) and the pulmonary type (SCCOPT). SCCOPT was first reported in 1992 by Eichhorn et al. (2). It typically affects postmenopausal women with a median age of 49 - 59 years $(4,7)$. The clinical manifestations are usually atypical, including abdominal pain, bloating, and abdominal mass. A few may suffer from irregular vaginal bleeding.

Pathological morphology shows that most of the tumors are small round cells, with few cytoplasm, hyperchromatic nuclei and unobvious nucleoli, arranged in sheets, nests, islands and trabeculae. Neuroendocrine markers such as neuron specific enolase (NSE), CD56, CgA and synaptophysin (Syn) are usually expressed $(6,8)$. In our case, CD56 and Syn were positive, and CgA was partially positive.

To date, literature reports about SCCOPT are relatively rare, and most of them are related to its pathological characteristics, treatment and prognosis, with only few literatures emphasizing on its imaging features. Hence, radiologists have insufficient understanding about it. Due to its similarity with epithelial ovarian cancers in clinical symptoms and laboratory tests, it is not easily distinguished pre- 


\begin{tabular}{ll}
\hline Table 1. Patients' Characteristics & \\
\hline & Details \\
\hline Sex & Female \\
\hline Age, $\mathbf{y}$ & 51 \\
\hline Symptom & Lower abdominal pain and distension \\
\hline Laboratory examination & CA125: 68.79 U/mL; CA199: 43.51 IU/mL; CEA: 15.87 ng/mL \\
\hline Imaging examinations & Ultrasound: a complex mass; MRI: heterogeneous lobulated mass with honeycomb-like intense enhancement \\
\hline Immunohistochemistry & CD56 (+); synaptophysin (+); chromogranin-A (+) \\
\hline Treatment & Surgical resection combined with postoperative chemotherapy \\
\hline
\end{tabular}

Abbreviations: CA, cancer antigen; CD, cluster of differentiation; CEA carcino-embryonic antigen; MRI, magnetic resonance imaging.

operatively. Based on our patient's pathological manifestations and previous literatures, we summarize the specific MRI findings of SCCOPT.

Approximately $25 \%$ - $50 \%$ of pure SCCOPT may involve bilateral ovaries (2). Most of the reported lesions are large in size and irregular in shape, with a maximum size of 30 $\mathrm{cm}$. The majority of SCCOPT is ill-defined with lobulated or nodular appearance. Most literatures (7, 9-11) report it as predominantly solid with focal hemorrhage and necrosis (as some cases did not mention whether they existed or not, the incidence could not be calculated), while some other cases present as solid cystic masses (12), with the cystic part mostly being mucus, significant necrosis and cystic degeneration. No cases of cystic SCCOPT have been reported. But when combined with other tumors (such as serous or myxoid tumors or mature cystic teratoma), it could be mainly cystic $(8,13)$. Calcification has not been mentioned in the literature. Based on previous studies (9, 14) and our case, SCCOPT features iso- to hypo-intense signal on T1WI, with spot-like high-signal when hemorrhage is present. On T2WI, the lesion shows mixed signals, mainly slightly higher or intermediate signals. T2WI FS of the solid component shows slightly higher signal with focal low signal, and the cystic part shows T2 isointense to water. DWI and ADC mapping show restricted diffusion in the solid component (hyperintense on DWI and hypointense on ADC mapping) due to hypercellularity of cancer cells and high nuclear-cytoplasmic ratio. On enhanced scan, the solid component of the tumor enhances distinctly and persistently (14), which is different from the fast-in and fast-out type of enhancement of ovarian epithelial cancer. As malignant tissue is generally separated by fibrous stripes of varying thickness $(2,10)$, SCCOPT has a characteristic honeycomb-like enhancement. Pelvic effusion occurs in more than half of patients. Lymphadenopathy, as well as peritoneal, omental and mesenteric metastases are also common. When coexisting with other tumors, SCCOPT's MRI manifestations are variable, which may lead to misdiagnosis. Moreover, it is difficult to distinguish with metastatic tumors when bilateral ovaries are involved.

Allowing for its rarity, SCCOPT remains a challenging tumor to treat. Standard primary surgical debulking is the treatment of choice, followed by adjuvant chemotherapy. There is no clear consensus for the optimal regime for postoperative chemotherapy because of the small number of patients and unavailability of follow-up data. Pelvic radiation remains enigmatic. Patients diagnosed with SCCOPT usually carry a very poor survival, generally dying rapidly within 2 years (6). There are also reports that indicate better prognosis when occurring with other tumors, which may be attributed to the small proportion of SCCOPT (15).

In our case, the imaging findings of the lesion are roughly consistent with the above literatures' description. Unfortunately, because of our insufficient understanding of SCCOPT, we failed to make a precise diagnosis. The main differential diagnosis includes ovarian epithelial cell carcinoma and thecoma-fibromas. Ovarian epithelial cell carcinoma shares a similar age of onset, clinical symptoms, and laboratory tests with our patient, as well as certain MR manifestations. MR appearance of ovarian epithelial carcinoma is a variable combination of cystic and solid components, with the solid component exhibiting apparent enhancement and restricted diffusion. Different pathological subtypes have different characteristics (16): serous cancers and clear cell cancers are predominantly unilocular cysts with intermediate signal papillary projections inside; mucinous tumors feature different signal intensities of locules based on variable content; and endometrioid tumors are associated with endometrial hyperplasia or frank endometrial carcinoma in up to one - third of cases. The MR characteristics of thecoma-fibromas are hypo-intensity on both $\mathrm{T} 1$ and $\mathrm{T} 2$ weighted images. After administration of gadolinium, these tumors have variable enhancement depending on the proportion of fibrous cells and thecal cells, with reports of negligible and avid enhancement $(17,18)$. Moreover, hormones secreted by thecal cells may cause en- 



Figure 1. A 51-year-old woman with lower abdominal pain and distension. Unenhanced pelvic MR images. MRI of the pelvis shows a heterogeneous lobulated mass with solid and cystic components originating from the right adnexa. A, Axial T1 weighted imaging (T1WI) reveals the lesion had iso- to hypo-intense signal; B and E, T2 weighted imaging with fat suppression (T2W FS) and T2W imaging show the solid component has slightly higher heterogeneous signal intensity, with hypointense foci; the cystic component has the same signal intensity as water; $C$ and D, Diffusion weighted imaging (DWI) and apparent diffusion coefficient (ADC) mapping exhibit restricted diffusion in the solid component; E, Moderate pelvic effusion is observed $(*) ; \mathrm{F}$, Endometrium is slightly thickened.

dometrial thickening, which was also seen in our patient.

In conclusion, when an ovarian tumor appears as a large, heterogeneous, complex and irregular mass, with the solid component exhibiting restricted diffusion on 

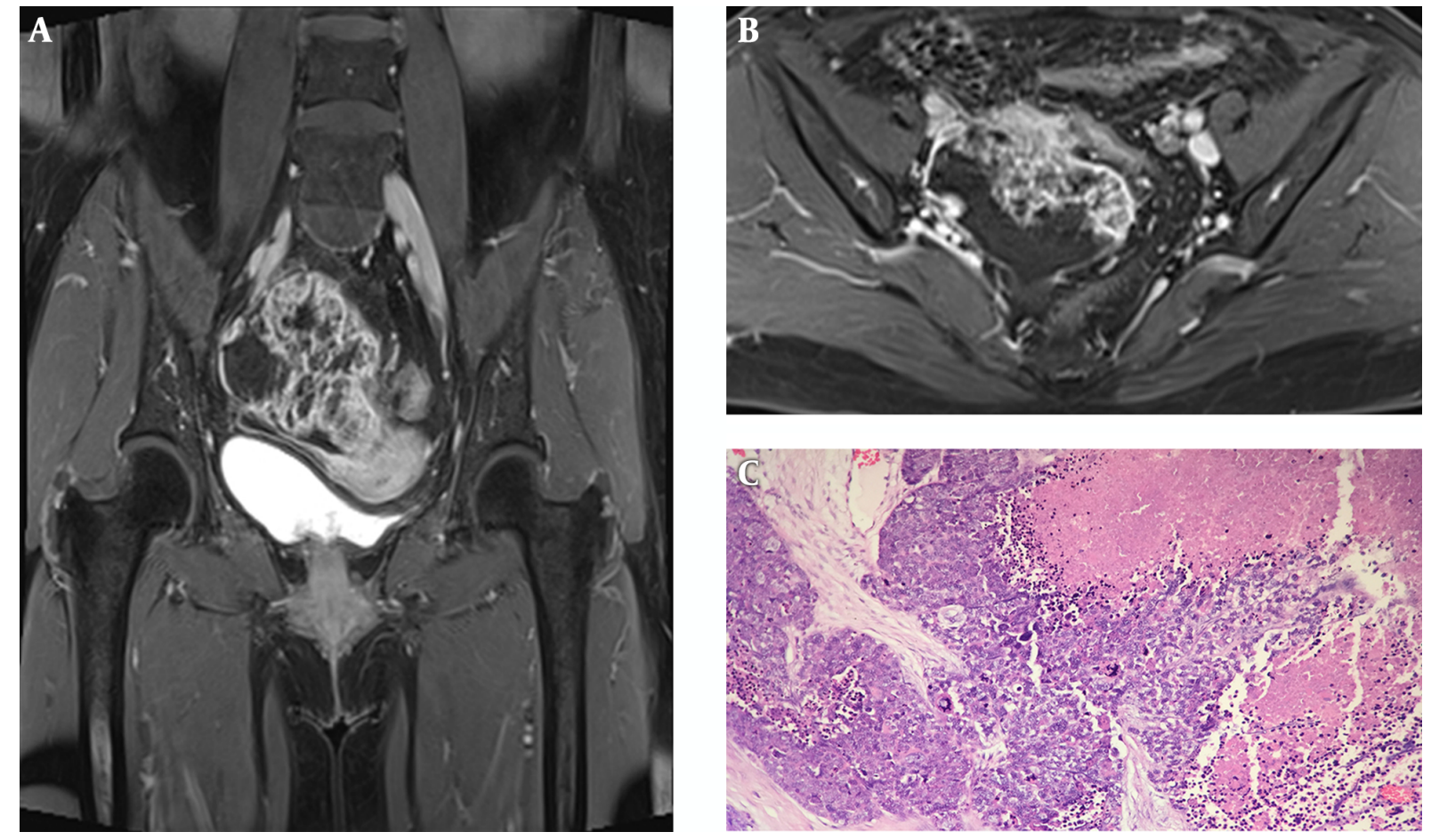

Figure 2. Contrast-enhanced pelvic MR images and pathology. A and B, The solid component of the lesion shows honeycomb-like intense enhancement. Irregular regions of different sizes without enhancement are also seen; C, The malignant neoplastic tissue consists classic closely packed small cells with scant cytoplasm, hyperchromatic nuclei, and obvious cell atypia. Malignant component is separated by abundant fibrous tissue, and large necrosis area is present. (Hematoxylin $\&$ eosin staining, $\times 200$ ).

DWI and honeycomb-like persistent enhancement, SCCOPT should be considered. But as the number of case reports is small, and the imaging findings has a lot of overlap with other ovarian tumors, more data are needed to better understand its specific manifestations.

\section{Footnotes}

Authors' Contributions: XD and PS drafted the manuscript, prepared the figures, and collected clinical data. YZ performed the histological examination. YZ and YY did selecting the case, supervising and editing the manuscript. ZP critically reviewed and revised the manuscript and supervised the study. All authors read and approved the final manuscript.

Conflict of Interests: The authors declare that they have no conflict of interests.

Ethical Approval: The manuscript has got ethical review exemption from Ethical Review Committee (ERC) of the authors' institution (Beijing Chaoyang Hospital, Capital Medical University) as case reports are exempted from review according to the Institutional Ethical Review Committee's Policy.
Funding/Support: This research did not receive any specific grant from funding agencies in the public, commercial, or not-for-profit sectors.

Informed Consent: Written consent was obtained from the patient for publishing the case (data and images).

\section{References}

1. Scully RE. Tumors of the ovary and maldeveloped gonads. Atlas of Tumor Pathology, 2nd series, Fascicle 16. Washington, DC: Armed Forces Institute of Pathology; 1979.39 p.

2. Eichhorn JH, Young RH, Scully RE. Primary ovarian small cell carcinoma of pulmonary type. A clinicopathologic, immunohistologic, and flow cytometric analysis of 11 cases. Am J Surg Pathol. 1992;16(10):926-38. doi: 10.1097/00000478-199210000-00002. [PubMed: 1384368].

3. Reed NS, Pautier P, Avall-Lundqvist E, Choi CH, du Bois A, Friedlander $\mathrm{M}$, et al. Gynecologic Cancer InterGroup (GCIG) consensus review for ovarian small cell cancers. Int J Gynecol Cancer. 2014;24(9 Suppl 3):S304. doi: 10.1097/IGC.0000000000000293. [PubMed: 25341577].

4. Atienza-Amores M, Guerini-Rocco E, Soslow RA, Park KJ, Weigelt B. Small cell carcinoma of the gynecologic tract: a multifaceted spectrum of lesions. Gynecol Oncol. 2014;134(2):410-8. doi: 10.1016/j.ygyno.2014.05.017. [PubMed: 24875120].

5. Yu JQ, Jia M, Li YM, Gao HW, Sun PL. Primary small-cell Carcinoma of the Ovary: A case report and literature review. J Clin Pathol Res. 2019;30(10):2321-8. doi:10.3978/j.issn.2095-6959.2019.10.035. 
6. Crowder S, Tuller E. Small cell carcinoma of the female genital tract. Semin Oncol. 2007;34(1):57-63. doi: 10.1053/j.seminoncol.2006.10.028. [PubMed: 17270667].

7. Yoshida Y, Kaneki E, Kijima M, Kodama K, Yamaguchi S, Yagi $\mathrm{H}$, et al. Two types of small cell carcinoma of the ovary: Two typical case reports. Gynecol Oncol Rep. 2018;25:125-30. doi: 10.1016/j.gore.2018.07.003. [PubMed: 30094313]. [PubMed Central: PMC6072903].

8. Grandjean M, Legrand L, Waterkeyn M, Baurain JF, Jadoul P, Donnez J, et al. Small cell carcinoma of pulmonary type inside a microinvasive mucinous cystadenocarcinoma of the ovary: a case report. Int J Gynecol Pathol.2007;26(4):426-31. doi: 10.1097/pgp.0b013e318039373a. [PubMed: 17885493].

9. Kira N, Takai N, Ishii T, Kai K, Nishida M, Nasu K, et al. Ovarian small cell carcinoma complicated by carcinomatous meningitis. Rare Tumors. 2012;4(2). e26. doi: 10.4081/rt.2012.e26. [PubMed: 22826783]. [PubMed Central: PMC3401154].

10. Kurasaki A, Sakurai N, Yamamoto Y, Taoka H, Takahashi K, Kubushiro K. Ovarian pulmonary-type small cell carcinoma: case report and review of the literature. Int J Gynecol Pathol. 2013;32(5):464-70. doi: 10.1097/PGP.ob013e31826d7ea8. [PubMed: 23896708].

11. Zhang JJ, Zhao WH. Research progress of primary small cell ovarian cancer. Prog Obstet Gynecol. 2013;22(7):602-3.

12. Tsolakidis D, Papanikolaou A, Ktenidis K, Pervana S. Primary ovarian small cell carcinoma of pulmonary type with enlarged paraaortic lymph node masses: a case report and review of the literature. Eur J Gynaecol Oncol. 2012;33(3):312-5. [PubMed: 22873108].

13. Rubio A, Schuldt M, Chamorro C, Crespo-Lora V, Nogales FF. Ovarian Small Cell Carcinoma of Pulmonary Type Arising in Mature Cys tic Teratomas With Metastases to the Contralateral Ovary. Int J Surg Pathol. 2015;23(5):388-92. doi: 10.1177/1066896915586254. [PubMed: 25990936].

14. Ren CC, Zhang XD, Zou Y, Lv BJ. MRI findings of ovarian small cell carcinoma in 3 cases. Chin J Radiol. 2017;51(11):875-6.

15. Qu LM, Zhong YP, Guo L, Ni JS. [Mucinous cystadenocarcinoma of ovary associated with neuroendocrine carcinoma (Primary ovarian pulmonary-type small cell carcinoma): report of a case]. Zhonghua Bing Li Xue Za Zhi. 2018;47(5):380-2. Chinese. doi: 10.3760/cma.j.issn.0529-5807.2018.05.015. [PubMed: 29783809].

16. Richard C; Semelka. Abdominal-Pelvic MRI. 3rd ed. Hoboken: John Wiley \& Sons, Inc; 2010. p. 1531-3.

17. Kitajima K, Kaji Y, Sugimura K. Usual and unusual MRI findings of ovarian fibroma: correlation with pathologic findings. Magn Reson Med Sci. 2008;7(1):43-8. doi: 10.2463/mrms.7.43. [PubMed: 18460848].

18. Schwartz RK, Levine D, Hatabu H, Edelman RR. Ovarian fibroma: findings by contrast-enhanced MRI. Abdom Imaging. 1997;22(5):535-7. doi: 10.1007/s002619900257. [PubMed: 9233896]. 